\title{
Analysis of Solution Residues by Glow Discharge Mass Spectrometry
}

\author{
Christopher M. Barshick, Douglas C. Duckworth, and David H. Smith \\ Analytical Chemistry Division, Oak Ridge National Laboratory, Oak Ridge, Tennessee, USA
}

\begin{abstract}
A technique for the analysis of microliter volumes of solution by glow discharge mass spectrometry (GDMS) has been successfully demonstrated. Cathode preparation involves mixing an aliquot of the sample solution with a pure conducting powder, followed by drying and pressing before conventional GDMS analysis. The analyte signal at the 100-ppm level was observed to be stable to better than $5 \%$ for the duration of the analysis (30-45 min). Internal and external reproducibilities were better than $5 \%$, and the ion signal intensity was linear with concentration over at least four orders of magnitude. Quantification was demonstrated by means of user-defined relative sensitivity factors. Relative standard deviations were better than $15 \%$ for the elements investigated, with no preconcentration of the analyte. (J Am Soc Mass Spectrom 1993, 4, 47-53)
\end{abstract}

$\mathrm{A}$ lthough the strong suit of glow discharge mass spectrometry (GDMS) is its ability to analyze solid samples directly, many instances arise where the sample of interest is already present in an aqueous medium. Traditionally, the analysis of these samples is accomplished by using atomic absorption spectroscopy (AAS) [1], inductively coupled plasma optical emission spectroscopy (ICP-OES) [2], or, more recently, inductively coupled plasma mass spectrometry (ICP-MS) [3-5]. There are instances, however, where the sample volume is too small for the conventional solution nebulization associated with these techniques, or the analyte concentration is already too low to allow dilution of the sample to a suitable volume; with these samples, graphite furnace AAS [6] or electrothermal vaporization inductively coupled plasma emission spectroscopy (ETV-ICP-OES) [7] is often used. With ETV-ICP-OES, detection limits of about $1 \mathrm{ng} / \mathrm{mL}$ for a $20-\mu \mathrm{L}$-solution volume have been reported [8]. Like its solution nebulization counterpart, ETV-ICPOES has given way to ETV-ICP-MS, with an improvement in both sensitivity and dynamic range [9].

One variation on the ETV theme is furnace atomic nonthermal excitation spectrometry (FANES); in this technique a thin solution residue is both atomized and excited in a graphite tube. Using FANES, Falk et al. [10-12] demonstrated superior detection limits compared with flameless AAS $(0.0007-16 \mu \mathrm{g} / \mathrm{L})$, as well as high dynamic range and multielement capability. A

Address reprint requests to Christopher M. Barshick, Analytical Chemistry Division, Oak Ridge National Laboratory, Oak Ridge, TN 37831-6375. complementary technique, furnace ionization nonthermal excitation spectrometry (FINES), has extended this work to include the detection of ionic emission [13], with the promise of mass spectrometric quantification and concurrent improvements in the analytical figures of merit.

The shortcoming of these thermal atomization approaches is the transient nature of the analyte signal. To obtain a large signal the analyte is often atomized very quickly. In some cases, this process lasts only a few seconds, during which time the resulting analyte cloud must be probed. In addressing this problem, several investigators have used the GD as an atomization source. In the GD, atomization is not thermal but is accomplished through cathodic sputtering. On the time scale for analyte detection, cathodic sputtering appears as a steady-state erosion of the solution residue and supporting medium. Redeposition and subsequent resputtering of a large fraction of the atoms [14], combined with the relatively slow sputtering rate (in milligrams per minute) of the GD [14], increase the time available for probing an analyte signal to several minutes. In the early 1970s, Daughtrey and Harrison [15], working with atomic emission, showed that boron could be determined in sub-parts per million concentrations from solution residues dried on commercially available hollow-cathode lamps. Although the largest analyte signal was observed during the first $30 \mathrm{~s}$, a measurable emission intensity above background was observed for up to $3 \mathrm{~min}$. Harrison and co-workers $[16,17]$ later extended their work to include mass spectrometric detection to improve sensitivity and provide multielement component analysis. More recently, GD 
analysis of solution residues has been explored in cupped cathodes [18], on tungsten wires [19], and on rhenium ribbons [20]. Jakubowski and Stuewer [21] have shown that dried solution residues, when sputtered off a GD cathode, can produce analytically useful signals even at low picogram concentrations. Concurrent with these GD studies, at least one group is investigating a similar technique for solution residue analysis in secondary ion mass spectrometry [22]. A1though all of these approaches have demonstrated some measure of success, the inherent problems associated with measuring transient ion signals still remain.

Recently, we have been evaluating new ways of preparing GD sample cathodes that permit the acquisition of data for several hours (instead of minutes). The results of these initial experiments are presented here.

\section{Experimental}

\section{Sample Preparation}

Two different GD cathode preparation techniques were used in this study. For all of the work described, the samples were prepared from residues of National Institute of Standards and Technology (NIST) standard stock solutions (SRM 3114, SRM 3128, SRM 3171, and SRM 3172). The first method involved pipetting 200 $\mu \mathrm{L}$ of solution into a polyethylene reservoir $3.5 \mathrm{~mm}$ in diameter $\times 20.3 \mathrm{~mm}$ deep. A silver electrode (99.999\%) was prepared by pressing $0.5 \mathrm{~g}$ of 325 -mesh silver powder at 15,000 psi to form a pin $1.5 \mathrm{~mm}$ in diameter $\times 20.0 \mathrm{~mm}$ long. This pin electrode was placed in the reservoir and the solvent allowed to evaporate at room temperature for $12 \mathrm{~h}$. A solid residue of the analyte was distributed on the surface of the GD electrode in a manner similar to drying the solution in a hollow cathode. A second cathode preparation technique was also investigated. This method involved pipetting 200 $\mu \mathrm{L}$ of solution into $1.0 \mathrm{~g}$ of $99.999 \%$ silver powder; the resulting slurry was then dried at $100^{\circ} \mathrm{C}$ for a minimum of $30 \mathrm{~min}$, mixed to ensure homogeneity, and pressed into a pin $1.5 \mathrm{~mm}$ in diameter $\times 20.0 \mathrm{~mm}$ in length.

\section{Mass Spectrometer}

A VG9000 GD mass spectrometer was used in this study (VG Elemental, Winsford, Cheshire, England). The system uses a reverse Nier-Johunson geometry double-focusing mass spectrometer. Faraday and Daly detectors provide a dynamic range in excess of $10^{9}$. The instrument nurmally uperates with an acceleration voltage of $8000 \mathrm{~V}$ and is capable of providing working resolutions of $4000-7000$ full width at half-maximum.

The discharge support gas for all of these studies was high-purity argon, further purified by an in-line heated active-metal getter system $\left(400^{\circ} \mathrm{C}\right)$. The liquid nitrogen-cooled "gallium" discharge cell was used; operating at about $-130^{\circ} \mathrm{C}$, this cell helps to reduce the interfering contributions of oxide and hydride species to the parts per billion level. The discharge voltage for all of these studies was typically $1000 \mathrm{~V}$ dc at a constant $2-\mathrm{mA}$ current. The ion source chamber pressure was $8 \times 10^{-5}$ mbar, and the cell pressure, although not directly measured, was estimated to be $7.5 \times 10^{-2}-0.75$ bar.

\section{Results and Discussion}

To evaluate our efforts in solution residue analysis by GDMS, we compared the two different methods of cathode preparation (surface deposition and bulk deposition). When one method was shown to be superior to the other, it was then characterized with respect to several analytical figures of merit. Internal and external reproducibilities were measured and quantification of a number of different elements evaluated.

\section{Comparison of Cathode Preparation Methods}

A 100-ppm lead working solution was prepared by dilution of a 10,000-ppm NIST SRM 3128 lead stock solution; GD cathodes were then prepared by the two different methods outlined previously. Because a typical GDMS analysis takes between 5 and $15 \mathrm{~min}$ (measuring between three and five elements for at least two naturally occurring and uninterfered isotopes), and at least three repetitive measurements are required for a reliable analysis, we chose to monitor the discharge for $45 \mathrm{~min}$ to gauge the effect of time on ion signal response. The analog signal for the Daly detector was output to a strip chart recorder, and the inn signal intensity was monitored from the time of discharge initiation. Figure 1 illustrates the ${ }^{208} \mathrm{~Pb}^{+}$signal intensity versus time, plotted in seconds for surface (Figure 1a) and bulk (Figure 1b) deposition. Uncertainty limits (1 SD) are indicated for each point. As anticipated, the ion signal in Figure 1a decays more rapidly during the first $300 \mathrm{~s}$ of the analysis than that in Figure $1 \mathrm{~b}$; in the former case, the bulk of the analyte material is concentrated on the surface of the silver electrode. Surprisingly, however, both curves reach a plateau after the first $5 \mathrm{~min}$ and remain relatively constant for the duration of the run. Although this implies that either cathode preparation method could be used, a closer examination of Figure 1 $\mathbf{a}$ and $\mathbf{b}$ indicates that the intensity from 300 to $1000 \mathrm{~s}$ varies by about $30 \%$ in Figure 1a, compared with only a $2 \%$ variation over the same region in Figure 1b. Another difference in the two cathodes is the ion intensity after equilibration; for the cathode in Figure 1a, with the solution deposited at the electrode surface, the ${ }^{208} \mathrm{~Pb}$ ion signal intensity is 30 times less than for the cathode in Figure 1b, where the solution is deposited in the electrode bulk. This disparity results because a few minutes of cathode sputtering serve to erode surface material and begin sampling 


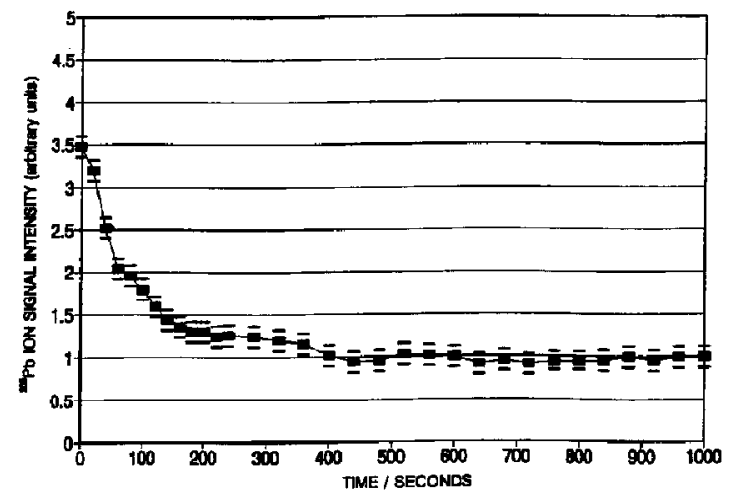

a

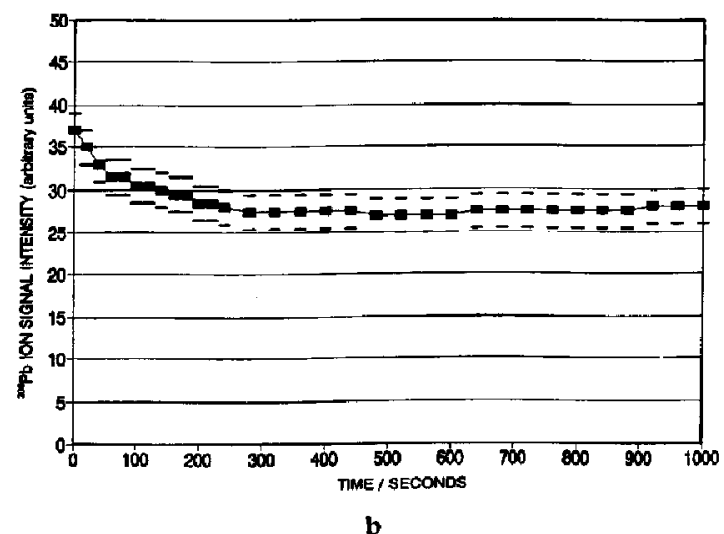

Figure 1. ${ }^{208} \mathrm{~Pb}$ ion signal versus time for (a) $200 \mu \mathrm{L}$ of a 100-ppm solution deposited on the surface of a silver electrode and (b) $200 \mu \mathrm{L}$ of a $100-\mathrm{ppm}$ solution deposited in the bulk of a silver electrode (see text). Time $t=0$ is the point of cathode initiation.

atoms from the bulk. Because only a small fraction of the analyte is absorbed into the bulk in the cathode in Higure la, it follows that the absolute signal intensity should be smaller than in the cathode in Figure 1b, where the solution is homogenized with the powdered electrode material. Physical loss during sample preparation and loading might also contribute to a smaller ion signal in the case of surface deposition; we believe these to be small, however.

The factors critical in the preparation of compacted powder electrodes are the homogeneity and particle size of the individual materials. It has been reported that particle diameters of $30-40 \mu \mathrm{m}$ or less may be necessary to ensure good precision and accuracy $[23,24]$. Winchester [25] observed that discharge stability is directly proportional to the average particle size and sample homogeneity. We believe that the relatively poor stability of our discharge from the surfacedeposited analyte is directly related to the nonuniform distribution of the lead at the cathode surface, as well as the inability of the GD to mix adequately the ana- lyte and matrix. This problem is lessened when bulk doping is used, because the entire sample, analyte and matrix, is mixed before pressing. It is also obvious that the manner in which we deposit the solution residue on the cathode surface is not very reproducible, either in the degree of coverage or the amount of deposition. Because both discharge stability and signal intensity will affect the precision of an analysis, we conclude that deposition of the solution material into the bulk of the cathode material is a superior method for analyzing solutions by GDMS. This methodology was used for all of the remaining investigations.

\section{Analytical Calibration Curve}

To determine the potential of a technique for quantification, it is necessary to evaluate several analytical figures of merit. One way to do this is by measuring the instrumental response for a range of concentrations. Because GDMS signal response in the analysis of solids has been shown to be linear over a broad range of elemental concentrations, a calibration curve is typically not used. Instead, relative sensitivity factors (RSFs) are determined from standard materials to correct for variations in elemental sensitivities and then applied to measurements made of unknown materials. The use of RSF values is favored because the difficulty in preparing a wide range of standard concentrations in solid form is alleviated. To see whether linearity holds for solution residue analysis, an analytical calibration curve was generated by accumulating data for five different cathodes ranging from $10 \mathrm{ppb}$ to 100 ppm in lead in the original solution; a pure silver cathode spiked with $200 \mu \mathrm{L}$ of distilled water was also analyzed. The ion intensity of each element of interest was then reduced by the appropriate amount corresponding to the blank signal. In this manner, the calibration sensitivity and detection limit were calculated. Five sequential measurements were made for each cathode to assess the run-to-run reproducibility. An average relative standard derivation value of $\pm 2 \%$ was calculated, indicating that the internal reproducibility is approaching bulk solid GDMS analysis $[26,27]$. Figure 2 is a blank-corrected $\log -\log$ calibration curve plotted as the ratio of the ${ }^{208} \mathrm{~Pb}^{+}$intensity to the ${ }^{107} \mathrm{Ag}^{+}$intensity versus lead concentration in parts per billion in the original solution. Because a calibration curve can only be linear until the analyte concentration falls below the detection limit, the four points indicated by the solid squares were used to calculate a linear regression [the fifth point (open square) obviously deviates from linearity]. The calibration sensitivity, defined as the slope of the calibration curve, is 0.775 , and the $y$ intercept is -8.76 . The broken line that runs horizontally from the $y$ axis to the calibration curve is from a point defined as the log of three times the standard deviation of the blank divided by the calibration sensitivity. After establishing this, a vertical 


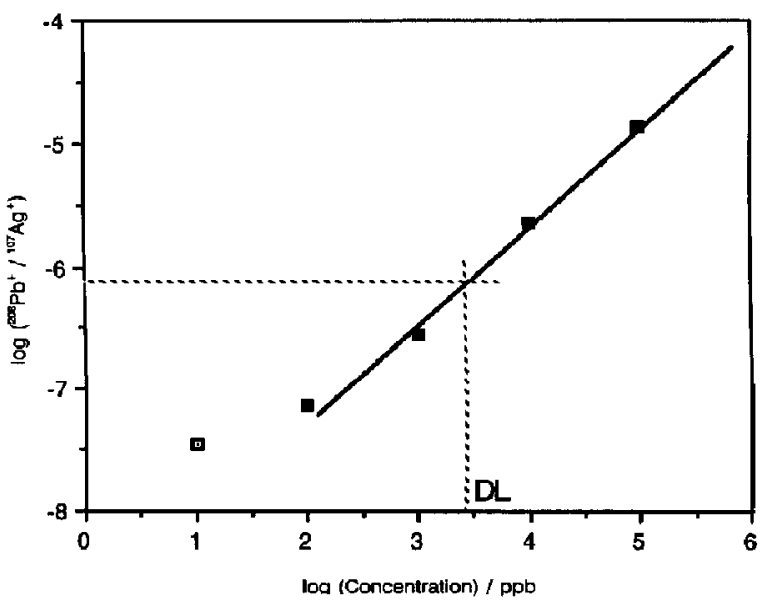

Figure 2. Blank-corrected calibration curve plotted as the log of the ratio of ${ }^{208} \mathrm{~Pb}^{+}$to ${ }^{107} \mathrm{Ag}^{+}$versus the log of the lead concentration in parts-per-billion (see text for explanation of symbols). DL, detection limit.

line is dropped to the point that corresponds to the log of some concentration (i.e., the detection limit) in parts per billion. For the data reported here, that value is ca. $2.5 \mathrm{ppm}$ in solution. Although this result is at least two orders of magnitude above published detection limits [28], these literature values were obtained using wellcharacterized solid standards that were well homogenized, and they refer to the bulk material and not to a concentration in solution. In making the solution-tosolid transition, it follows that if $200 \mu \mathrm{L}$ (ca. $200 \mathrm{ng}$ ) of a 1.0-ppm solution is mixed with $1.0 \mathrm{~g}$ of the matrix powder, a fivefold dilution of the solution has been introduced by the solid. The net result is a smaller absolute concentration in the bulk than in solution (ca. 0.2 for this example and $0.5 \mathrm{ppm}$ at the detection limit).

\section{Sample-to-Sample Reproducibility}

The sample-to-sample reproducibility was measured by preparing four separate cathodes from solutions, 1.0 ppm each in lead. Using cathode 1 , the ion signal intensities for the ${ }^{107} \mathrm{Ag},{ }^{109} \mathrm{Ag},{ }^{206} \mathrm{~Pb},{ }^{207} \mathrm{~Pb}$, and ${ }^{208} \mathrm{~Pb}$ ions were recorded for six trials. An average RSF value was calculated for lead, relative to silver, by the relationship:

$$
\operatorname{RSF}_{x}=\left(I_{r} / C_{r}\right) /\left(I_{x} / C_{x}\right)
$$

where $I_{x}$ is the average signal intensity for species $\mathbf{x}$ (lead, isotopically corrected) at a known concentration $C_{x}$, and $I_{x}$ is the average signal intensity of the reference $r$ (silver, isotopically corrected) at a known concentration $C_{r}$. The RSF value for lead at $1.0 \mathrm{ppm}$, assuming a silver concentration of $100 \%$, was 0.621 . This RSF value was then used for the analysis of lead in cathodes 2-4. The three values obtained for cathodes 2,3 , and 4 were $1.11,0.90$, and 0.95 , respectively. The RSD of the RSF values for the three cathodes was \pm 0.11 , indicating good sample-to-sample reproducibility and good agreement with literature values [26].

\section{Mass Spectra}

Figure 3 shows two different regions of the mass spectrum obtained for a solution that was $100 \mathrm{ppm}$ in both copper and lead. Working solutions of these concentrations were made from NIST SRM 3114 and SRM 3128 stock solutions, respectively. In Figure $3 a$, the major peaks result from the copper isotopes at $m / z 63$ and 65 , along with peaks at $m / z 68$ and 69 , presumably $\mathrm{ArN}_{2}^{+}$and $\mathrm{ArN}_{2} \mathrm{H}^{+}$, polyatomic ions formed from trace levels of nitric acid and water. Minor components at $m / z 62,64,66$, and 67 are unidentified polyatomic interferences, although $m / z 64,66$, and 67 could be due to $\mathrm{Zn}^{+}$. Full-scale ion intensity for the spectrum is $5.0 \times 10^{-15} \mathrm{~A}$.
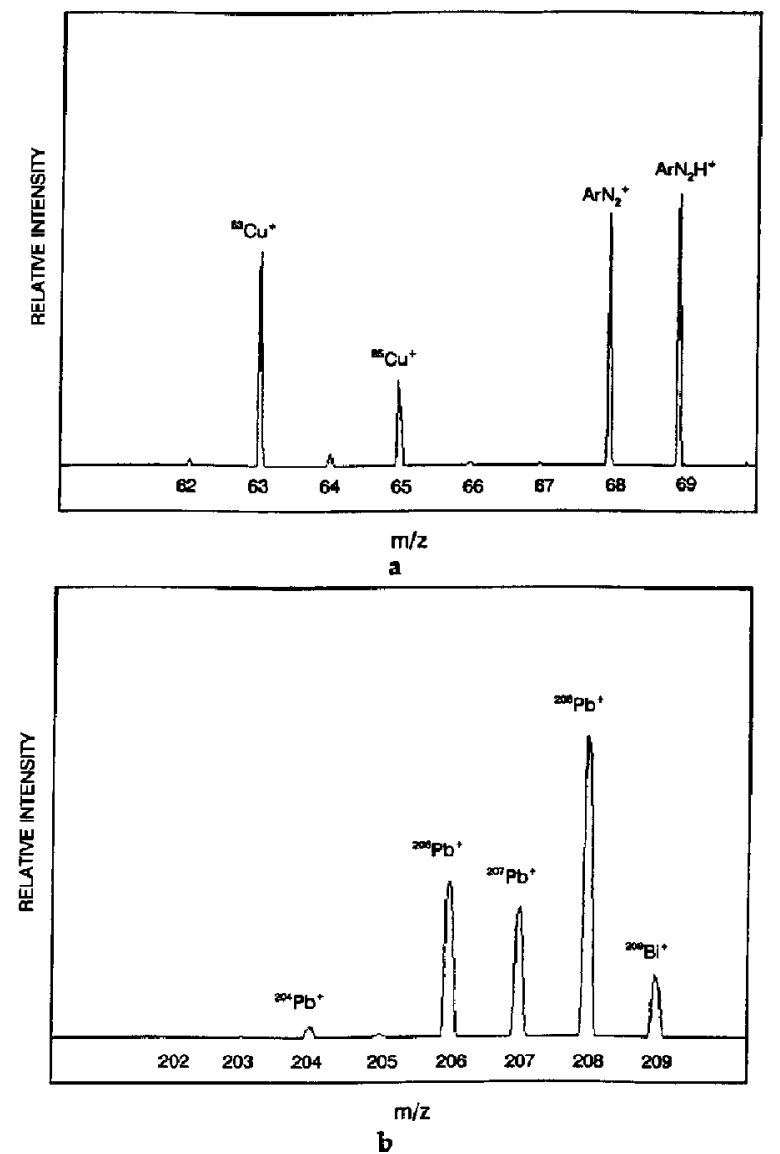

Figure 3. Glow discharge mass spectrum of a silver cathode doped with $200 \mu \mathrm{L}$ of a solution that is $100 \mathrm{ppm}$ in copper and $100 \mathrm{ppm}$ in lead, over the mass range (a) $6070 \mu$, and (b) $200-210 \mu(1000 \mathrm{~V} \mathrm{dc} ; 2.0 \mathrm{~mA}$; ca. 1 torr argon $)$. 
A potential problem in the analysis of solutions is the formation of molecular ions in the form of an electronegative species from the solvent combining with a metal atom. The seriousness of this problem varies with the solvent system and ionization conditions, among other parameters. In this study such species were observed under moderate resolution conditions at the 100-ppm level. These were of particular concern for the case of copper at $m / z 63$ and 65 , where these contaminants undoubtedly led to errors in the measured isotopic ratios. The contaminants are still unidentified, but their presence underscores the importance of careful spectral examination to ensure that measured peaks reliably represent the species of interest.

Figure $3 \mathrm{~b}$ shows the $200-210-\mu$ mass range of the same spectrum acquired in Figure $3 a$. The lead isotopes at $m / z 204,206,207$, and 208, as well as the ${ }^{209} \mathbf{B i}$ isotope (a known contaminant in the matrix at less than $4 \mathrm{ppm}$ ) are clearly visible. Also observed are interferences at $m / z 203$ and 205, possibly due to $\mathrm{Tl}^{+}$. Isotope ratios agree with expected values to about $\pm 3.0 \%$ for the ${ }^{206} \mathrm{~Pb},{ }^{207} \mathrm{~Pb}$, and ${ }^{208} \mathrm{~Pb}$ ions; an obvious interference at mass 204 is detected although not resolved. Full-scale ion intensity for the spectrum is $2.0 \times 10^{-15} \mathrm{~A}$.

\section{Quantitative Analysis}

Because accurate elemental quantification relies on good relative sensitivity factors, it would appear that obtaining a wide variety of standard reference materials from which to make these measurements would become increasingly important; however, unlike some elemental mass spectrometric techniques (e.g., secondary ion mass spectrometry), bulk solids GDMS sensitivity factors typically differ from element to element by a factor of less than 10 . It is therefore tempting to conclude that a "standard" set of RSF values can be used for every analysis, and indeed both VG Elemental and others [29] have published RSF values that have been widely used.

Relative sensitivity factors do, however, show a systematic variation depending on the plasma conditions (pressure, power, etc.), as well as the source geometry, ion optics, and the sample matrix $[29,30]$. It is therefore common to determine sensitivity values before each analysis to ensure better accuracy. This situation is not unlike solution-based elemental mass spectrometry where a blank and a standard(s) are analyzed before the unknown analysis; however, it is somewhat more time-consuming than using standard RSF values.

Because our matrix is different from that of a bulk solid material, we determined our own RSF values. Two cathodes were prepared containing $200 \mu \mathrm{L}$ of an NIST SRM 3171 multielement mix A standard solution, $200 \mu \mathrm{L}$ of an NIST SRM 3172 multielement mix B standard solution, and $1.0 \mathrm{~g}$ of silver power. The compacted sample was analyzed for aluminum, beryllium, cadmium, chromium, iron, magnesium, nickel, potassium, sodium, barium, calcium, copper, lead, sele-

Table 1. Analyte concentrations in a glow discharge cathode prepared from two NIST standard reference solutions using standard RSFs

\begin{tabular}{lrcc}
\hline Element & $\begin{array}{c}\text { Concentration } \\
(\mu \mathrm{g} / \mathrm{mL})\end{array}$ & $\begin{array}{c}\text { GDMS standard } \\
\mathrm{RSF}^{\mathrm{a}}\end{array}$ & $\begin{array}{c}\text { GDMS Concentration } \\
(\mu \mathrm{g} / \mathrm{mL})\end{array}$ \\
\hline SRM 3171 & $100.0 \pm 0.5$ & 0.719 & $150.8 \pm 44.6$ \\
Aluminum & $10.0 \pm 0.1$ & 0.657 & $8.1 \pm 1.6$ \\
Beryllium & $100.0 \pm 0.5$ & 2.657 & $25.2 \pm 2.4$ \\
Cadmium & $100.0 \pm 0.5$ & 0.637 & $34.6 \pm 5.1$ \\
Chromium & $100.0 \pm 0.5$ & 0.286 & $39.8 \pm 3.2$ \\
Iron & $100.0 \pm 0.5$ & 0.369 & $25.6 \pm 2.5$ \\
Magnesium & $100.0 \pm 0.5$ & 0.423 & $25.5 \pm 2.5$ \\
Manganese & $100.0 \pm 0.5$ & 0.440 & $25.9 \pm 0.8$ \\
Nickel & $500.0 \pm 2.5$ & - & $438.4 \pm 98.2$ \\
Potassium & $100.0 \pm 0.5$ & 0.714 & $83.5 \pm 20.4$ \\
Sodium & $10.0 \pm 0.1$ & & $11.6 \pm 1.8$ \\
SRM 3172 & $10.0 \pm 0.1$ & - & $27.8 \pm 6.2$ \\
Barium & $100.0 \pm 0.5$ & 0.163 & $26.5 \pm 1.6$ \\
Calcium & $100.0 \pm 0.5$ & 1.417 & $19.2 \pm 1.6$ \\
Copper & $500.0 \pm 2.5$ & 0.626 & $166.6 \pm 14.1$ \\
Lead & $10.0 \pm 0.1$ & 0.886 & $12.2 \pm 1.4$ \\
Selenium & $100.0 \pm 0.5$ & - & $17.2 \pm 1.3$ \\
Strontium & Zinc & 1.560 & \\
Zinc & & & \\
\hline
\end{tabular}

${ }^{a}$ Relarive sensitivity factor (RSF) values relative to silver and abtained from ref. 27 (see text); GDMS. glow discharge mass spectrometry. 
nium, strontium, and zinc using the ${ }^{27} \mathrm{Al},{ }^{9} \mathrm{Be},{ }^{114} \mathrm{Cd}$, ${ }^{52} \mathrm{Cr}$ and ${ }^{53} \mathrm{Cr},{ }^{56} \mathrm{Fe}$ and ${ }^{57} \mathrm{Fe},{ }^{55} \mathrm{Mn},{ }^{60} \mathrm{Ni},{ }^{39} \mathrm{~K},{ }^{23} \mathrm{Na}$, ${ }^{138} \mathrm{Ba},{ }^{42} \mathrm{Ca},{ }^{63} \mathrm{Cu},{ }^{208} \mathrm{~Pb},{ }^{77} \mathrm{Se},{ }^{88} \mathrm{Sr}$, and ${ }^{66} \mathrm{Zn}$ isotopes, respectively. The results, shown in Table 1 as GDMS concentration, are the average of three trials, T1-T3, using RSF values relative to silver as determined by Vieth and Huneke [29]. The average relative error was ca. $60 \%$, ranging from $16 \%$ for barium to $178 \%$ for calcium. Using the intersity measurements from T1-T3, we calculated new RSF values, shown in Table 2 (third column). The sample was reanalyzed and these new RSF values applied to trials T4-T6; the results are shown in Table 2 (fourth column). Relative errors averaged ca. $19 \%$, with a low of ca. $3 \%$ for aluminum and a high of $50 \%$ for sodium, a significant improvement over trails T1-T3. Finally, a second set of RSF values was calculated using the intensity measurements for all six trials, T1-T6. The second cathode, previously unstudied, was analyzed and the cumulative RSF values from trials T1-T6 applied to the results. The average relative error decreased to $14 \%$, ranging from $2 \%$ to $30 \%$. Although these results are encouraging, the wide variations in RSFs (ranging to factors of upward of 150) and modest relative standard deviations indicate that more methods development is necessary to reach the level of precision and accuracy that bulk solids GDMS and solution-based ICP-MS possess. In particular, we believe that it will be necessary to address further some possible sources of contamination, interelement variations due to interfer- ences, and still unaccounted for sputtering phenomena that caused some of the results to fall too low and some too high.

\section{Conclusions}

Glow discharge mass spectrometry solution residue analysis has been shown to be complementary to bulk solid GDMS. Microliter volumes of solutions can be analyzed by mixing and drying them with a conducting matrix before electrode formation. This method, because it is solution based, is well suited for analyzing samples where conventional GDMS cathode preparation has shortcomings ( $<1 \mathrm{mg}$ of sample). Although the results reported here were obtained from solution residues, it offers the possibility that it will be viable for analyzing small samples originating as solids by means of similar techniques. Our preliminary results indicate that detection limits are on the order of 2.5 $\mathrm{ppm}$ in a $200-\mu \mathrm{L}$ volume of solution. This technique is complementary to ICP-MS and AAS. It may prove valuable for analyzing high brine and particulate samples directly, without dilution; this possibility awaits experimental verification. As with bulk solids analysis, quantification for a wide range of elements is accomplished through the use of NIST standard reference materials and user-defined RSFs. Unlike conventional solids GDMS analysis, isotope dilution is available when the best accuracy is required. Because residual acid and water are present in the electrode, care must

Table 2. Analyte concentrations in a glow discharge cathode prepared from two NIST standard reference solutions using user-defined RSFs

\begin{tabular}{|c|c|c|c|}
\hline Element & $\begin{array}{c}\text { Concentration } \\
(\mu \mathrm{g} / \mathrm{mL})\end{array}$ & $\begin{array}{l}\text { GDMS measured } \\
\text { RSF }^{*}\end{array}$ & $\begin{array}{c}\text { GDMS Concentration } \\
(\mu \mathrm{g} / \mathrm{mL})\end{array}$ \\
\hline \multicolumn{4}{|l|}{ SRM 3171} \\
\hline Aluminum & $100.0 \pm 0.5$ & 0.690 & $96.6 \pm 23.5$ \\
\hline Beryllium & $10.0 \pm 0.1$ & 1.002 & $8.9 \pm 2.4$ \\
\hline Cadmium & $100.0 \pm 0.5$ & 12.323 & $141.1 \pm 1.5$ \\
\hline Chromium & $100.0 \pm 0.5$ & 2.293 & $89.5 \pm 19.2$ \\
\hline Iron & $100.0 \pm 0.5$ & 0.827 & $86.2 \pm 14.2$ \\
\hline Magnesium & $100.0 \pm 0.5$ & 1.736 & $115.4 \pm 20.0$ \\
\hline Manganese & $100.0 \pm 0.5$ & 2.001 & $92.8 \pm 6.3$ \\
\hline Nickel & $100.0 \pm 0.5$ & 2.008 & $94.7 \pm 9.3$ \\
\hline Potassium & $500.0 \pm 2.5$ & 1.422 & $452.74 \pm 51.9$ \\
\hline Sodium & $100.0 \pm 0.6$ & 1.336 & $166.2 \pm 17.5$ \\
\hline \multicolumn{4}{|l|}{ SRM 3172} \\
\hline Barium & $10.0 \pm 0.1$ & 1.040 & $14.0 \pm 6.5$ \\
\hline Calcium & $10.0 \pm 0.1$ & 0.074 & $11.7 \pm 1.0$ \\
\hline Copper & $100.0 \pm 0.5$ & 6.559 & $90.4 \pm 12.3$ \\
\hline Lead & $100.0 \pm 0.5$ & 3.895 & $88.9 \pm 3.2$ \\
\hline Selenium & $500.0 \pm 2.5$ & 3.202 & $775.5 \pm 110.1$ \\
\hline Strontium & $10.0 \pm 0.1$ & 0.972 & $6.6 \pm 2.0$ \\
\hline Zinc & $100.0 \pm 0.5$ & $1 \uparrow .192$ & $119.8 \pm 11.1$ \\
\hline
\end{tabular}

Relative sensitivity factor (RSF) values relative to silver and obtained from the intensity measure ments of trials T1-T3 (see text); GDMS, glow discharge mass spectrometry. 
be taken in determining interferences; this is especially important for isotope ratio applications where high mass resolution may be necessary.

\section{Acknowledgment}

We thank L. C. Satterfield of the Analytical Chemistry Division, Oak Ridge National Laboratory, for providing the NIST standard reference solutions.

\section{References}

1. Slavin, W. Anal. Chem. 1991, 63, 924A.

2. Boumans, P. W. J. M., Ed. Inductively Coupled Plasma Emission Spectroscopy; Wiley: New York, 1987; Part I.

3. Olesik, J. W. Anal. Chem. 1991, 63, 12A.

4. Collantes, E, R.; Dunn III, W. J. Appl. Spectrosc. 1991, 45, 1537.

5. Ekimoff, D.; Van Norstrand, A. M.; Mowers, D. A. Appl. Spectrosc. 1989, 43, 1252.

6. L'Vov, B. V. Anal. Chem. 1991, 63, 924A.

7. Sturgeon, R. F.; Willie, S. N.; Luong, V.; Berman, S. S. Anal. Chem. 1990, 62, 2370 .

8. Kumamaru, T,; Okamoto, Y.; Matsuo, H. Appl. Spectrosc. $1987,41,918$.

9. Shen, W. L.; Caruso, J. A.; Fricke, F. L.; Satzger, R. D. I. Anal. At. Spectrom. 1990, 5, 451.

10. Falk, H.; Hoffman, E.; Jaeckel, I.; Lüdke, C. Spectrochim. Acta 1979, 34B, 333 .

11. Falk, H.; Hoffman E.; Lüdke, C. Spectrochim. Acta B 1981, 36, 767.
12. Falk, H.; Hoffman, E.; Lüdke, C. Spectrachim. Acta B 1984, 39, 283.

13. Ditrich, K.; Eismanu, G.; Fuchs, H. J. Anal. At. Spectrom. 1988, 3,459 .

14. Barshick, C. M. Ph.D dissertation, University of Virgina, 1990.

15. Daughtrey, E. H.; Harrison, W. W. Anal. Chim. Acta 1973, 67, 253.

16. Daughtrey, E. H.; Harrison. W. W. Anal. Chem. 1975, 47, 1024.

17. Donohue, D. L.; Harrison, W. W. Anal. Chem. 1975, 47, 1528.

18. Keefe, R. B. Ph.D. dissertation, University of Virginia, 1983.

19. Ma, Yi-Zai; Harrison, W. W., unpublished results.

20. Ratliff, P. H.; Harrison, W. W., unpublished results.

21. Jakubowski, N.; Stuewer, D.; Toelg, G. Spectrochim. Acta B 1991, 46, 155.

22. Chia, V. K. F.; Bleiler, R. I.; Sams, D. B.; Craig, A. Y.; Odom, R. W. Appl. Phys. Lett. 1991, 59, 2567.

23. McDonald, D. C. Anal. Chem., 1977, 49, 1336.

24. Caroli, S.; Alimonti, A.; Zimmer, K. Spectrochim. Acta B 1983, $38,625$.

25. Winchester, M. R. Ph.D. dissertation, Clemson University, 1990.

26. Harrison, W. W.; Bentz, B. L. Prog. Anal. Spectrosc, 1988, 11, 53.

27. Jakuboski, N.; Stuewer, D.; Vieth, W. Fresenius Z. Anal. Chem. 1988, 331, 145.

28. Sanderson, N. E.; Hall, E.; Clark, J.; Charlambous, P.; Hall D. Mikrochim. Acta 1987, I, 275.

29. Vieth, W.; Huneke, J. C. Spectrochim. Acta B 1991, 46, 137.

30. King, F. L. Ph.D. dissertation, University of Virginia, 1989. 